Tôhoku Math. Journ. 31 (1979), 301-307.

\title{
NOTES ON THE CLASS FIELD TOWERS OF CYCLIC FIELDS OF DEGREE $l$
}

\author{
Teruo TAKeUChI
}

(Received July 31, 1978, revised October 5, 1978)

1. Introduction. Let $l$ be a rational odd prime. Let $k$ be an algebraic number field of finite degree, $K / k$ be a cyclic extension of degree $l$, and $\mathfrak{p}_{1}, \cdots, \mathfrak{p}_{t}$ denote the prime ideals in $k$ ramified in $K$. Then, as is well-known, the genus number of $K$ with respect to $k$ is given by $h(k) l^{t-1} /\left(E_{k}: E_{k} \cap N_{K / k} K^{\times}\right)$, where $h(k)$ denotes the class number of $k$ and $E_{k}$ denotes the group of units in $k$. (See, for instance, [1].) Though the genus number of $K / k$ is determined uniquely by $\mathfrak{p}_{1}, \cdots, \mathfrak{p}_{t}$, this expression does not give explicitly the relations between the genus number of $K / k$ and the prime ideals $\mathfrak{p}_{1}, \cdots, \mathfrak{p}_{t}$.

Let $p_{i}$ denote the rational prime contained in $\mathfrak{p}_{i}$ and assume $p_{i} \equiv 1$ $\bmod l$ for $i=1, \cdots, t$. In this note, we shall first show that the genus number of $K / k$ is calculated by the subgroup of $\operatorname{Gal}\left(k\left(\zeta, E_{k}^{1 / l}\right) / k\right)$ which is generated by the decomposition groups of prime divisors of $\mathfrak{p}_{i}$ in $k\left(\zeta, E_{k}^{1 / l}\right)$, where $\zeta$ is a primitive $l$-th root of unity.

Next, we shall apply the above result together with the Čebotarev density theorem to the class field tower problem to show the existence of fields which satisfy some properties.

Let $k / \boldsymbol{Q}$ be a cyclic extension of degree $\boldsymbol{l}$ and $p_{1}, \cdots, p_{t}$ denote the primes in $\boldsymbol{Q}$ ramified in $k$. It is well-known that the $\boldsymbol{l}$-class field tower of $k$ is infinite if $t$ is sufficiently large. (Cf. [5].) Moreover, we know by a result of Y. Furuta [2] that if $p_{1}, \cdots, p_{t}$ are prime to $l$ and $8 \leqq t$, then the $l$-class field tower of $k$ is infinite. On the other hand, if $t=1$, then $l \nmid h(k)$ so the $l$-class field tower of $k$ is finite. In this note, we consider the case when $t=2$ and obtain the following theorems.

THEOREM 1. Let $l$ and $p_{1}$ be odd primes with $13 \leqq l$ and $p_{1} \equiv 1$ mod $l$. Then there exist infinitely many primes $p_{2}$ which satisfy the following conditions:

(i ) $p_{2} \equiv 1 \bmod l$,

(ii) the l-class field tower of $k$ is infinite for every cyclic extension $k / \boldsymbol{Q}$ of degree $\boldsymbol{l}$ in which only $p_{1}$ and $p_{2}$ are ramified.

THEOREM 2. Let $l$ be an odd prime and $p_{1}$ be an odd prime with 
$p_{1} \equiv 1 \bmod \boldsymbol{l} . \quad$ Let $k_{1} / \boldsymbol{Q}$ be the cyclic extension of degree $\boldsymbol{l}$ in which only $p_{1}$ is ramified. Assume that $4(2+l) \leqq h\left(k_{1}\right)$, where $h\left(k_{1}\right)$ is the class number of $k_{1}$. Then there exist infinitely many primes $p_{2}$ which satisfy the following conditions:

(i) $p_{2} \equiv 1 \bmod l$,

(ii) the l-class field tower of $k$ is finite but the class field tower of $k$ is infinite for every cyclic extension $k / Q$ of degree $\boldsymbol{l}$ in which only $p_{1}$ and $p_{2}$ are ramified.

2. The genus numbers of cyclic extensions. Let $l$ be a rational odd prime. Let $k$ be an algebraic number field of finite degree and let $K$ be a cyclic extension of degree $l$ over $k$. For an ideal $a$ in $k$, let $I(\mathfrak{a})$ denote the group of ideals in $k$ prime to a, $P(\mathfrak{a})$ the group of principal ideals in $I(\mathfrak{a})$, and $P_{a}$ the ray mod $a$. Let $\mathfrak{p}_{1}, \cdots, \mathfrak{p}_{t}$ be the prime ideals in $k$ ramified in $K$, and put $c=\mathfrak{p}_{1} \cdots \mathfrak{p}_{t}$. Let $K^{(1)}$ be the Hilbert class field of $K$. Then, by definition, the genus field of $K / k$ is the maximal abelian extension of $k$ included in $K^{(1)}$. If $c$ is prime to $l$, then the conductor of $K / k$ is c. So the following lemma is easily proved.

Lemma 1. Let the notations be as above. Assume $\mathrm{c}$ is prime to $\mathrm{l}$. Then the genus field of $K$ over $k$ is the class field corresponding to the ideal class group $I(\mathrm{c}) / P(\mathrm{c})^{l} P_{\mathrm{c}}$.

Next, we shall study the order of $P(\mathfrak{c}) / P(c)^{l} P_{c}$. Let $P^{*}(k)$ be the set of prime ideals $\mathfrak{p}$ in $k$ which are prime divisors of rational primes $p$ with $p \equiv 1 \bmod l$. Let $\zeta$ be a primitive $l$-th root of unity and put $k_{0}=k(\zeta)$. Let $\bar{k}_{0}=k_{0}\left(E_{k}^{1 / l}\right)$, where $E_{k}$ is the group of units in $k$. Then $\bar{k}_{0}$ is a Galois extension of $k$. Put $G=\operatorname{Gal}\left(\bar{k}_{0} / k_{0}\right)$ and $\bar{E}=E_{k} / E_{k}^{l}$. Then we see that

$$
k_{0}^{l} E_{k} / k_{0}^{l} \approx \bar{E}=E_{k} / E_{k}^{l} .
$$

Indeed, for $\varepsilon$ in $E_{k}$ if $\varepsilon$ is in $k_{0}^{l}$, then $\varepsilon$ is in $k^{l}$, therefore $\varepsilon$ is in $E_{k}$. Hence we can develop the Kummer theory for $\bar{E}$, as follows. Let $Z_{t}$ denote the group of $l$-th roots of unity. We define $\langle\rangle:, G \times \bar{E} \rightarrow Z_{l}$ by $\langle\sigma, \bar{\varepsilon}\rangle=\sigma\left(\varepsilon^{1 / l}\right) / \varepsilon^{1 / l}$. Then $\langle$,$\rangle is a non-degenerate bilinear form. Let$ $\mathfrak{c}=\mathfrak{p}_{1} \cdots \mathfrak{p}_{t}$ be a product of distinct primes in $P^{*}(k)$ and let $\bar{E}(\mathfrak{c})=$ $\left(E_{k} \cap k(c)^{l} k_{c}\right) / E_{k}$, where $k(c)=\{\alpha \in k \mid(\alpha) \in P(c)\}$ and $k_{c}=\left\{\alpha \in k \mid(\alpha) \in P_{c}\right\}$. Let $\bar{G}(c)$ be the group of elements orthogonal to $\bar{E}(c)$ with respect to $\langle$,$\rangle .$

Let $\mathfrak{P}_{i}$ be a prime divisor of $\mathfrak{p}_{i}$ in $\bar{k}_{0}$ and let $G\left(\mathfrak{\beta}_{i}\right)$ be the decomposition group of $\mathfrak{P}_{i}$. Then $G\left(\mathfrak{P}_{i}\right) \subset G$, since $\mathfrak{p}_{i}$ is completely decomposed in $k_{0}$. Therefore, by the Kummer theory, $G\left(\mathfrak{P}_{i}\right)$ is a normal subgroup of 
Gal $\left(\bar{k}_{0} / k\right)$. Hence $G\left(\mathfrak{P}_{i}\right)$ depends only on $\mathfrak{p}_{i}$. Thus we may write $G\left(\mathfrak{p}_{i}\right)$ instead of $G\left(\mathfrak{P}_{i}\right)$.

Under the above notations, we have the following propositions.

Proposition 1.

(i ) If $\mathfrak{p}$ is in $P^{*}(k)$, then $\bar{G}(\mathfrak{p})=G(\mathfrak{p})$.

(ii) If $c_{1}$ and $c_{2}$ are relatively prime, then $\bar{G}\left(c_{1} c_{2}\right)=\bar{G}\left(c_{1}\right) \bar{G}\left(c_{2}\right)$.

(iii) If $\sigma$ is an automorphism of $\bar{k}_{0}$ which induces an automorphism on $k$, then $\bar{G}(\sigma c)=\sigma \bar{G}(\mathfrak{c}) \sigma^{-1}$.

Proof. (i) Let $\varepsilon$ be a unit of $k$. If $\varepsilon$ is in $k_{p} k(\mathfrak{p})^{l}$, then the equation $X^{l} \equiv \varepsilon \bmod \mathfrak{p}$ has an integral solution in $k$. Therefore $\mathfrak{p}$ is completely decomposed in $k\left(\varepsilon^{1 / l}\right)$, hence $\varepsilon$ is in the decomposition field $\bar{k}_{0}^{G(\mathfrak{p})}$ of $\mathfrak{p}$. Conversely, if $\varepsilon$ is in $\bar{k}_{0}^{G(\mathfrak{p})}$, then $\varepsilon$ is in $k_{\mathfrak{p}} k(\mathfrak{p})^{l}$. For (ii) it suffices to note that $\bar{E}\left(c_{1} c_{2}\right)=\bar{E}\left(c_{1}\right) \bar{E}\left(c_{2}\right)$. (iii) It is easy to see that $\varepsilon \in \bar{E}(\sigma c)$ if and only if $\sigma^{-1} \varepsilon \in \bar{E}(\mathfrak{c})$, and $\langle\tau, \varepsilon\rangle=1$ if and only if $\left\langle\sigma^{-1} \tau \sigma, \sigma^{-1} \varepsilon\right\rangle=1$. Thus the assertion follows immediately.

Proposition 2. Let $\mathfrak{c}=\mathfrak{p}_{1} \cdots \mathfrak{p}_{t}$ be a product of distinct primes in $P^{*}(k)$. Then \# $\left(P(\mathfrak{c}) / P(\mathfrak{c})^{l} P_{\mathrm{c}}\right)=\boldsymbol{l}^{t} / \#(\bar{G}(\mathrm{c}))$.

Proof. We first note that

$$
P(\mathrm{c}) / P(\mathrm{c})^{l} P_{\mathrm{c}} \approx k(\mathrm{c}) / E_{k} k(\mathrm{c})^{l} k_{\mathrm{c}} .
$$

Since $k(c) / k(c)^{l} k_{c}$ is an elementary abelian group of rank $t$, we see that

$$
\begin{aligned}
\#\left(P(\mathrm{c}) / P(\mathrm{c})^{l} P_{\mathrm{c}}\right) & =\#\left(k(\mathrm{c}) / E_{k} k(\mathrm{c})^{l} k_{\mathrm{c}}\right) \\
& =\#\left(k(\mathrm{c}) / k(\mathrm{c})^{l} k_{\mathrm{c}}\right) / \#\left(E_{k} k(\mathrm{c})^{l} k_{\mathrm{c}} / k(\mathrm{c})^{l} k_{\mathrm{c}}\right) \\
& =\boldsymbol{l}^{t} / \#\left(E_{k} k(\mathrm{c})^{l} k_{\mathrm{c}} / k(\mathrm{c})^{l} k_{\mathrm{c}}\right) .
\end{aligned}
$$

On the other hand,

$$
E_{k} k(\mathrm{c})^{l} k_{\mathrm{c}} / k(\mathrm{c})^{l} k_{\mathrm{c}} \approx E_{k} /\left(E_{k} \cap k(\mathrm{c})^{l} k_{\mathrm{c}}\right)
$$

and $\#\left(E_{k} /\left(E_{k} \cap k(\mathfrak{c})^{l} k_{c}\right)\right)=l^{r} / \bar{E}(\mathfrak{c})=\#(\bar{G}(\mathfrak{c}))$, where $r$ is the $l$-rank of $E_{k}$. Hence we have \#(P(c)/P(c)l$\left.P_{\mathrm{c}}\right)=\boldsymbol{l}^{t} / \#(\bar{G}(\mathrm{c}))$.

3. Proof of Theorem 1. Let $l$ be an odd prime. Let $p_{1}$ be an odd prime with $p_{1} \equiv 1 \bmod \boldsymbol{l}$, and let $k_{1} / \boldsymbol{Q}$ be a cyclic extension of degree $\boldsymbol{l}$ in which only $p_{1}$ is ramified, where $\boldsymbol{Q}$ is the field of rationals. Let $\sigma$ be a generator of $\mathrm{Gal}\left(k_{1} / \boldsymbol{Q}\right)$ and let $\odot$ be the maximal order of $\boldsymbol{Q}(\zeta)$, where $\zeta$ denotes a primitive $l$-th root of unity. Let $E_{1}$ be the group of units in $k_{1}$. Then $E_{1} / E_{1}^{\prime}$ is a module over $Z[\sigma]$. Moreover, since $N\left(E_{1}\right)=\{ \pm 1\} \subset E_{1}, E_{1} / E_{1}^{\prime}$ is also a module over $Z[\sigma] /\left(1+\sigma+\cdots+\sigma^{l-1}\right) Z[\sigma]$, where $N$ denotes the norm map of $k_{1}$ to $\boldsymbol{Q}$. Therefore we can consider $E_{1} / E_{1}^{\prime}$ as a module over $\odot$ by $\sigma \mapsto \zeta$. 
LEMMA 2. $E_{1} / E_{1}^{l}$ is O-isomorphic to $O / I^{l-1}$, where $\mathfrak{l}$ is the prime divisor of $l$ in $O$.

Proof. Since $l \nmid h\left(k_{1}\right)$, the cyclotomic units in $k_{1}$ generate $E_{1} / E_{1}^{l}$. On the other hand, the cyclotomic units in $k_{1}$ are conjugate to each other. Therefore $E_{1} / E_{1}^{l}$ is a principal $\mathcal{O}^{-}$-module. Since the rank of $E_{1} / E_{1}^{l}$ is $l-1$, we see that $E_{1} / E_{1}^{l}$ is isomorphic to $\mathscr{O} / \mathfrak{l}^{l-1}$.

Proposition 3. Let $p_{1}$ be an odd prime with $p_{1} \equiv 1 \bmod l$, and let $r$ be a natural number with $1 \leqq r \leqq l-1$. Then there exist infinitely many odd primes $p_{2}$ which satisfy the following conditions:

(i ) $p_{2} \equiv 1 \bmod l$,

(ii) the genus number of $k_{1} k_{2}$ with respect to $k_{1}$ is $h\left(k_{1}\right) l^{r}$, where $k_{2} / \boldsymbol{Q}$ is the cyclic extension of degree $\boldsymbol{l}$ in which only $p_{2}$ is ramified.

Proof. Let $M=\left(\mathscr{O} \pi^{s}+\boldsymbol{Z} \pi^{s-2}+\boldsymbol{Z} \pi^{s-3}+\cdots+\boldsymbol{Z} \pi+\boldsymbol{Z}\right) / \mathfrak{I}^{\boldsymbol{I - l}}$ be a subgroup of $O / \mathfrak{l}^{l-l}$, where $\pi=\zeta-1$ and $s=l-1-r$. Then the maximal

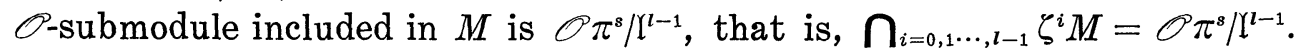
Indeed, let $\alpha=a_{s-2} \pi^{s-2}+\cdots+a_{0}$ be in $\bigcap \zeta^{i} M$, where $a_{i} \in \boldsymbol{Z} / \boldsymbol{l} \boldsymbol{Z}$. Then $\zeta \alpha$ is in $M$. Hence we see that $a_{s-2}=0$, since $\zeta \alpha=a_{s-2} \pi^{s-1}+\left(a_{s-2}+\right.$ $\left.a_{s-3}\right) \pi^{s-2}+\cdots+\left(a_{1}+a_{0}\right) \pi+a_{0}$. Similarly, $\alpha=0$ since $\zeta^{j} \alpha \in M$ for $j=2, \cdots, l-1$.

Now, let $E_{M}$ be the subgroup of $E_{1} / E_{1}^{l}$ corresponding to $M$ by the isomorphism in Lemma 2. Let $k_{0}=k_{1}(\zeta), \bar{k}_{0}=k_{0}\left(E_{1}^{1 / l}\right)$, and $k_{0}(M)=k_{0}\left(E_{M}^{1 / l}\right)$. Then $\bar{k}_{0} / k_{0}(M)$ is a cyclic extension. Let $\tau$ be a generator of Gal $\left(\bar{k}_{0} / k_{0}(M)\right)$. Then we see by the Čebotarev density theorem that there exist infinitely many prime ideals $\Re_{2}$ in $\bar{k}_{0}$, unramified over $\boldsymbol{Q}$, such that the Frobenius symbol $\left[\mathfrak{F}_{2}, \bar{k}_{0} / \boldsymbol{Q}\right]=\tau$. Let $p_{2}=\mathfrak{F}_{2} \cap \boldsymbol{Q}$ and $\mathfrak{p}_{2}=\mathfrak{\beta}_{2} \cap k_{1}$. Then $p_{2} \equiv 1 \bmod \boldsymbol{l}$, hence $\mathfrak{p}_{2} \in P^{*}\left(k_{1}\right)$. Since $k_{1}$ is Galois over $\boldsymbol{Q}, p_{2}$ is completely decomposed in $k_{1}$. Let $\sigma$ be a generator of $\operatorname{Gal}\left(k_{1} / \boldsymbol{Q}\right)$. Then $p_{2}=\mathfrak{p}_{2}\left(\sigma \mathfrak{p}_{2}\right) \cdots\left(\sigma^{l-1} \mathfrak{p}_{2}\right)$ in $k_{1}$. Since the prime ideals in $k_{1}$ ramified in $k_{1} k_{2}$ are $\mathfrak{p}_{2},\left(\sigma \mathfrak{p}_{2}\right), \cdots,\left(\sigma^{l-1} \mathfrak{p}_{2}\right)$, the genus field of $k_{1} k_{2} / k_{1}$ is the class field over $k_{1}$ corresponding to the ideal class group $I\left(p_{2}\right) / P\left(p_{2}\right)^{l} P_{p_{2}}$ of $k_{1}$. Hence the genus number is $\#\left(I\left(p_{2}\right) / P\left(p_{2}\right)^{\prime} P_{p_{2}}\right) / \boldsymbol{l}$. On the other hand, $G\left(\mathfrak{p}_{2}\right)=\operatorname{Gal}\left(\bar{k}_{0} / k_{0}(M)\right)$ and $G\left(\sigma^{i} \mathfrak{p}_{2}\right)=\operatorname{Gal}\left(\bar{k}_{0} / k_{0}\left(\zeta^{i} M\right)\right)$ for $i=1, \cdots, l-1$. Therefore by Proposition $1 \bar{G}\left(p_{2}\right)=G\left(\mathfrak{p}_{2}\right) \cdots G\left(\sigma^{l-1} \mathfrak{p}_{2}\right)=$ $\operatorname{Gal}\left(\bar{k}_{0} / k_{0}\left(\cap \zeta^{i} M\right)\right)$. Hence $\#\left(\bar{G}\left(p_{2}\right)\right)=l^{l-1} / \#\left(\cap \zeta^{i} M\right)=l^{l-1-(l-1-s)}=l^{i-1-r}$. Thus by Proposition 2 we see that

$$
\begin{aligned}
\#\left(I\left(p_{2}\right) / P\left(p_{2}\right)^{l} P_{p_{2}}\right) & =h\left(k_{1}\right) \#\left(P\left(p_{2}\right) / P\left(p_{2}\right)^{l} P_{p_{2}}\right) \\
& =h\left(k_{1}\right) l^{l} / l^{l-1-r}=h\left(k_{1}\right) l^{r+1} .
\end{aligned}
$$

This proves the proposition.

Proof of THEOREM 1. Let $p_{2}$ be a prime satisfying the conditions 
in Proposition 3 for $r=l-1$. Let $k / Q$ be a cyclic extension of degree $l$ in which only $p_{1}$ and $p_{2}$ are ramified. Then $k_{1} k_{2} / k$ is an unramified cyclic extension of degree $l$. Let $L$ be the maximal $l$-extension of $k_{1}$ included in the genus field of $k_{1} k_{2} / k_{1}$. Then $L$ is the class field over $k_{1}$ corresponding to $I\left(p_{2}\right) / I\left(p_{2}\right)^{l} P_{p_{2}}$. Now, we apply [2, Theorem 3] to this field $L$. Since the $l$-rank of $\operatorname{Gal}\left(L / k_{1}\right)$ is $l$ and $13 \leqq l$, we see that

$$
2+2(l-1+l+1)^{1 / 2} \leqq l-\operatorname{rank}\left(\operatorname{Gal}\left(L / k_{1}\right)\right) .
$$

Thus the $l$-class field tower of $L$ is infinite and the $l$-class field tower of $k$ is also infinite.

REMark. Let $p_{2}$ be a prime satisfying the conditions in Proposition 3 for $r=1$. Let $\sigma$ be a generator of $\mathrm{Gal}\left(K / k_{1}\right)$, where $K=k_{1} k_{2}$. Then we can consider the $l$-Sylow subgroup $M_{K}$ of the ideal class group of $K$ as a module over $\mathcal{O}$ by $\sigma \mapsto \zeta$. Since the $l$-part of the genus number of $K / k_{1}$ is $l, M_{K}$ is, as is seen by [4 I, Theorem 1], O-isomorphic to O/le, where $e$ is a natural number. Let $\tau$ be a generator of Gal $\left(K / k_{2}\right)$. Then $\tau$ operates on $M_{K}$ and hence on $O / l^{e}$ as an automorphism. Since $\sigma$ and

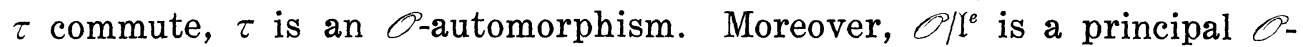
module. Therefore $\tau$ is represented by a unit $\alpha$ in O/fe. Since $\tau^{l}=1$, $\alpha$ is of the following form; $\alpha=1+a_{1} \pi+\beta \bmod l^{e}$, where $\beta$ is in $\mathfrak{l}^{2}, a_{1}$ is an integer with $0 \leqq a_{1} \leqq l-1$, and $\pi=\zeta-1$. Let $j$ be a natural number such that $j \not \equiv 0 \bmod l$ and $j+a_{1} \not \equiv 0 \bmod l$. Let $\pi^{\prime}=\alpha \zeta^{j}-1$. Since $\alpha \zeta^{j}=\alpha(1+\pi)^{j}=1+\left(j+a_{1}\right) \pi+\gamma \pi^{2} \bmod \mathfrak{l}^{e}, \pi^{\prime}$ is in $\mathfrak{l}$ but not in $\mathfrak{l}^{2}$. Therefore $\left(\mathscr{O} / \mathbb{I}^{e}\right) / \pi^{\prime}\left(\mathscr{O} / \mathbb{l}^{e}\right) \approx \mathscr{O} / \mathrm{l}$. Thus we see that $M_{K} / M_{K}^{o-1} \approx \mathcal{O} / \mathfrak{l}$ for $\rho=\tau \sigma^{j}$ in $\operatorname{Gal}(K / Q)$. Let $k$ be the fixed field of $\rho$. Then $k / Q$ is a cyclic extension of degree $l$ in which only $p_{1}$ and $p_{2}$ are ramified. Therefore the $\boldsymbol{l}$-Sylow subgroup $M_{k}$ of the ideal class group of $k$ is a module over $\mathcal{O}$ by $\sigma^{\prime} \mapsto \zeta$, where $\sigma^{\prime}$ is a generator of $\mathrm{Gal}(k / \boldsymbol{Q})$, and it is $\mathcal{O}_{\text {- }}$ isomorphic to $O / \mathbb{R}^{r}$ for a natural number $r$. Since $K / k$ is an unramified cyclic extension of degree $\boldsymbol{l}$, we see that $\#\left(M_{K} / N_{K / k} M_{K}\right)=l$ and $M_{K} / M_{K}^{\rho-1} \approx$ $N_{K / k} M_{K} \subset M_{k}$. Hence we have that $M_{k} \approx O / \mathbb{I}^{2}$. On the other hand, we know by [3, Proposition VI. 6] (see also [4 I, Corollary to Theorem 3]) that if $M_{k} \approx O / I^{2}$ for some cyclic extension $k / Q$ of degree $\boldsymbol{l}$ in which only $p_{1}$ and $p_{2}$ are ramified, then $M_{k^{\prime}} \approx \mathcal{O} / \mathbb{I}^{2}$ for every cyclic extension $k^{\prime} / \boldsymbol{Q}$ of degree $\boldsymbol{l}$ in which only $p_{1}$ and $p_{2}$ are ramified.

Thus we have the following.

For any odd prime $p_{1}$ with $p_{1} \equiv 1 \bmod \boldsymbol{l}$, there exist infinitely many primes $p_{2}$ which satisfy the following conditions:

(i) $p_{2} \equiv 1 \bmod l$,

(ii) $M_{k} \approx \mathcal{O} / \mathbb{1}^{2}$ for every cyclic extension $k / Q$ of degree $\boldsymbol{l}$ in which 
only $p_{1}$ and $p_{2}$ are ramified.

4. Proof of Theorem 2. Let $p_{1}$ and $k_{1}$ be as in 3 . Let $K$ be the Hilbert class field of $k_{1}, K_{0}=K(\zeta)$, and $\bar{K}_{0}=K_{0}\left(E_{K}^{1 / l}\right)$.

Proposition 4. Let the notations be as above. Then there exist infinitely many primes which satisfy the following conditins:

(i) $p_{2} \equiv 1 \bmod l$.

(ii) $\boldsymbol{l} \| h(k)$, i.e., $\boldsymbol{l} \mid h(k)$ and $\boldsymbol{l}^{2} \nmid h(k)$, for every cyclic extension $k / \boldsymbol{Q}$ of degree $l$ in which only $p_{1}$ and $p_{2}$ are ramified.

(iii) $l$-rank $(\operatorname{Gal}(L / K)) \geqq h\left(k_{1}\right)$, where $L$ is the genus field of $K k$ with respect to $K$.

Proof. Since $K / \boldsymbol{Q}$ is Galois, $\bar{K}_{0} / \boldsymbol{Q}$ is Galois. Let $\sigma$ be an element in $\mathrm{Gal}\left(\bar{K}_{0} / \boldsymbol{Q}\right)$ such that $\sigma^{l}=1$ and that $\sigma \notin \mathrm{Gal}\left(\bar{K}_{0} / K_{0}\right)$. Such $\sigma$ certainly exists. Indeed, the inertia group of a prime divisor of $p_{1}$ in $\bar{K}_{0}$ is a cyclic group of order $l$, and is not included in Gal $\left(\bar{K}_{0} / K_{0}\right)$. Then, by the Čebotarev density theorem, we see that there exist infinitely many unramified primes $\mathfrak{F}_{2}$ in $\bar{K}_{0}$ such that the Frobenius symbol $\left[\mathfrak{F}_{2}, \bar{K}_{0} / \boldsymbol{Q}\right]=\sigma$. Let $p_{2}=\boldsymbol{Q} \cap \mathfrak{P}_{2}$ and $\mathfrak{p}_{2}=K \cap \mathfrak{P}_{2}$. Since $p_{2}$ is completely decomposed in $Q(\zeta)$, it follows that $p_{2} \equiv 1 \bmod l$ and that $\mathfrak{p}_{2}$ is in $P^{*}(K)$. On the other hand, $\sigma$ generates $\operatorname{Gal}\left(k_{1} / Q\right)$. Indeed, since $\boldsymbol{l} \nmid h\left(k_{1}\right)$ and $K_{0} / k_{1}$ is Galois, $\operatorname{Gal}\left(\bar{K}_{0} / K_{0}\right)$ is the unique $l$-Sylow subgroup of $\operatorname{Gal}\left(\bar{K}_{0} / k_{1}\right)$. Hence $p_{2}$ is not decomposed in $k_{1}$ and $p_{2}$ is non $l$-th power residue $\bmod p_{1}$. Therefore, by the genus theory, the $l$-Sylow subgroup $M_{k}$ of the ideal class group of $k$ is a cyclic group of order $l$. Thus we have $l \| h(k)$. Since $p_{2}$ is not decomposed in $k_{1}$, it follows that $p_{2}$ is a principal prime ideal in $k_{1}$. Hence $p_{2}$ is completely decomposed in $K / k_{1}$, say $p_{2}=\mathfrak{p}_{2,1} \cdots \mathfrak{p}_{2, t}$, where $\mathfrak{p}_{2,1}=\mathfrak{p}_{2}$ and $t=h\left(k_{1}\right)$. Since $\sigma \notin \mathrm{Gal}\left(\bar{K}_{0} / K_{0}\right)$ and $\mathrm{Gal}\left(\bar{K}_{0} / K_{0}\right)$ is normal, every element conjugate to $\sigma$ is not contained in Gal $\left(\bar{K}_{0} / K_{0}\right)$. Hence $\mathfrak{p}_{2,1} \cdots \mathfrak{p}_{2, t}$ are completely decomposed in $\bar{K}_{0} / K$. Therefore by Proposition 1

$$
\bar{G}\left(p_{2}\right)=\bar{G}\left(\mathfrak{p}_{2,1} \cdots \mathfrak{p}_{2, t}\right)=G\left(\mathfrak{p}_{2,1}\right) \cdots G\left(\mathfrak{p}_{2, t}\right)=\{1\} .
$$

Thus by Proposition 2 we see that $\#\left(P\left(p_{2}\right) / P\left(p_{2}\right)^{l} P_{p_{2}}\right)=l^{t}$. Moreover, only $\mathfrak{p}_{2,1}, \cdots, \mathfrak{p}_{2, t}$ are ramified in $K k / K$, since only $p_{2}$ is ramified in $k_{1} k / k_{1}$. Therefore the genus field $L$ of $K k / K$ is the class field over $K$ corresponding to the ideal class group $I\left(p_{2}\right) / P\left(p_{2}\right) P_{p_{2}}$ of $K$. Hence we have that

$$
\begin{aligned}
l-\operatorname{rank}(\operatorname{Gal}(L / K)) & =l-\operatorname{rank}\left(I\left(p_{2}\right) / P\left(p_{2}\right)^{l} P_{p_{2}}\right) \\
& \geqq t=h\left(k_{1}\right) .
\end{aligned}
$$

This completes the proof.

Proof of TheOREM 2. Let $p_{2}$ be a prime satisfying the conditions in 
Proposition 4. Then $l \| h(k)$, hence the $l$-class field tower of $k$ is finite. Let $L$ be the genus field of $K k$ with respect to $K$. Now, we apply [2, Theorem 3] to this $L$. We first note that $h\left(k_{1}\right) \geqq 4(2+l)$ implies $h\left(k_{1}\right) \geqq$ $2+2\left(l h\left(k_{1}\right)-1+h\left(k_{1}\right)+1\right)^{1 / 2}$. On the other hand, $l$-rank $(\mathrm{Gal}(L / K)) \geqq h\left(k_{1}\right)$. Hence we have that

$$
\begin{aligned}
l \text {-rank }(\operatorname{Gal}(L / K)) & \geqq 2+2\left(\operatorname{lh}\left(k_{1}\right)-1+h\left(k_{1}\right)+1\right)^{1 / 2} \\
& \geqq 2+2\left(l \text {-rank }\left(E_{K}\right)+t+1\right)^{1 / 2} .
\end{aligned}
$$

Thus the $l$-class field tower of $L$ is infinite. Since $L / K k$ and $K k / k$ are unramified abelian extensions, the class field tower of $k$ is infinite. This proves our theorem.

REMARK. In the case $l=2$, an argument similar to Theorem 2 holds.

\section{REFERENCES}

[1] Y. Furuta, The genus field and genus number in algebraic number fields, Nagoya Math. J., 29 (1967), 281-285.

[2] Y. Furuta, On class field towers and the rank of ideal class group, Nagoya Math. J., 48 (1972), 147-157.

[3] G. Gras, Sur les $\ell$-classes d'ideaux dans les extensions cyclique relative de degré premier ל, Ann. Inst. Fourier 23, 3 (1973), 1-48; ibid. 23, 4 (1973), 1-44.

[4] T. TAKEUCHI, On the structure of $p$-class groups of certain number fields I, II, Sci. Rep. Niigata Univ. Series A 14 (1977), 25-33; ibid. 15 (1978), 35-42.

[5] P. RoquetTe, On class field towers, in Algebraic Number Theory, 231-249, Academic Press, New York, 1968.

Department of Mathematics

Faculty of General Education

NigGata UNIVERSITY

Nitgata, 950-21 JAPAN 
